\title{
A New View of Fuzzy Ordered Semigroups
}

\author{
Hidayat Ullah Khan ${ }^{1 *}$, Asghar Khan2, Faiz Muhammad Khan ${ }^{3}$, Amir Khan ${ }^{4}$, Muhammad Taj ${ }^{5}$
}

${ }^{1}$ Department of Mathematics, University of Malakand, Pakistan,

${ }^{2}$ Department of Mathematics, Abdul Wali Khan University Mardan, Pakistan

${ }^{3,4}$ Department of Mathematics and Statistics, University of Swat, Pakistan

${ }^{5}$ Department of Mathematics, University of Azad Jammu and Kashmir Muzaffarabad, Pakistan

* Corresponding author: hidayatullak@yahoo.com

\section{A b s t r a ct}

Fuzzy semigroup theory concentrates on theoretical aspects, but also includes applications in the areas of fuzzy coding theory, fuzzy finite state machines, and fuzzy languages. In this paper, we introduce the

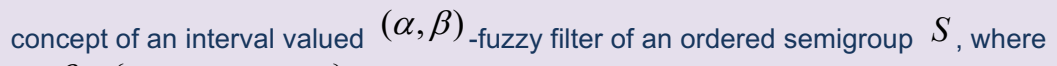
$\alpha, \beta \in\{\in, \mathrm{q}, \in \vee \mathrm{q}, \in \wedge \mathrm{q}\}$ with $\alpha \neq \in \wedge \mathrm{q}$. Since the concept of an interval valued $(\in, \in \vee \mathrm{q})_{\text {-fuzzy filter is }}$ an important and useful generalization of ordinary interval valued fuzzy filter, we discuss some fundamental aspects of an interval valued $(\in, \in \vee q)_{\text {-fuzzy filters. An interval valued }}(\in, \in \vee q)$-fuzzy filter is a generalization of the existing concept of an interval valued fuzzy filter. We discuss the concept of an interval

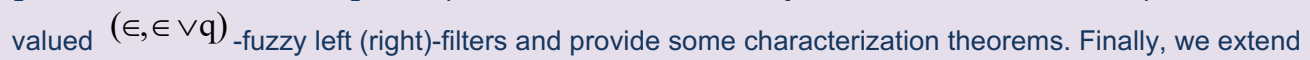
the concept of an interval valued fuzzy subgroup with thresholds to the concept of an interval valued fuzzy left (right)-filter with thresholds of $S$.

Keywords: Interval valued fuzzy filter; Interval valued $(\alpha, \beta)_{\text {-fuzzy filter; Interval valued }}(\in, \in \vee q)_{\text {-fuzzy }}$ filter; Interval valued $(\in, \in \vee \mathrm{q})_{\text {-fuzzy (left) right filter. }}$

\section{ARTICLE INFORMATION}

$\begin{array}{ll}\text { Received: } & 17 \text { February } 2018 \\ \text { Revised: } & 21 \text { June } 2018\end{array}$

Accepted: 10 November 2018

DOI:

10.31580/ojst.v1i1.150

(C) Readers Insight Publication

\section{INTRODUCTION}

The idea of a quasi-coincidence of a fuzzy point with a fuzzy set, which is mentioned in $(1,2)$, played a vital role to generate some different types of fuzzy subgroups. It is worth pointing out that Bhakat and Das (see (1)) gave the concepts of $(\alpha, \beta)$-fuzzy subgroups by using the belongs to" relation $(\in)$ and quasi-coincident with" relation (q) between a fuzzy point and a fuzzy subgroup, and introduced the concept of an $(\in, \in \vee q)$-fuzzy subgroup. In particular, $(\in, \in \vee q)$. fuzzy subgroup is an important and useful generalization of Rosenfeld's fuzzy subgroup (26). It is now natural to investigate similar type of generalizations of the existing fuzzy subsystems of other algebraic structures. Mordeson et al. in (24) presented an up to date account of fuzzy sub-semigroups and fuzzy ideals of a semigroup. The book concentrates on theoretical aspects, but also includes applications in the areas of fuzzy coding theory, fuzzy finite state machines, and fuzzy languages. Basic results on fuzzy subsets, semigroups, codes, finite state machines, and languages are reviewed and introduced, as well as certain fuzzy ideals of a semigroup and advanced characterizations and properties of fuzzy semigroups. With this objective in view, Kuroki (20) introduced the notion of fuzzy bi-ideals in semigroups. Jun and Song (see (11)) discussed general forms of fuzzy interior ideals in semigroups, also see (15). Kazanci and Yamak introduced the concept of a generalized fuzzy bi-ideal in semigroups (12) and gave some properties of fuzzy bi-ideals in terms of $(\in, \in \vee \mathrm{q})$-fuzzy bi-ideals. Jun et al. (see (9)) gave the concept of a generalized fuzzy bi-ideal in ordered semigroups and characterized regular ordered semigroups in terms of this notion. Davvaz et al. used the idea of generalized fuzzy sets in several algebraic structures and introduced different generalized fuzzy subsystems e.g., (see $(3-8,16))$. In (23), Ma et al. introduced the concept of a generalized fuzzy filter of $R_{0}$-algebra and provided some properties in terms of this notion, also see (22). Many other researchers used the idea of generalized fuzzy sets and gave several characterizations results in different branches of algebra see references.

The concept of a fuzzy filter in ordered semigroups was first introduced by Kehayopulu and Tsingelis in (14), where some basic properties of fuzzy filters and prime fuzzy ideals were discussed. In 1975, Zadeh (29) introduced the important concept of interval valued fuzzy subset on which the values of the membership functions are intervals of numbers instead of the numbers alone. In (30), Zeng and $\mathrm{Li}$ gave a kind of method to describe the entropy of interval valued fuzzy set based on its similarity measure and discussed their relationship between the similarity measure and the entropy of the interval valued fuzzy sets in detail.

In mathematics, an ordered semigroup is a semigroup together with a partial order that is compatible with the semigroup operation. Ordered semigroups have many applications in the theory of sequential machines, formal languages, computer arithmetics, design of fast address and error-correcting codes. A theory of fuzzy generalized sets on ordered semigroups can be developed. Using the idea of a quasi- 
coincidence of a fuzzy point with a fuzzy set, the concept of an interval valued $(\alpha, \beta)$-fuzzy filter in an ordered semigroup is introduced.

Our aim in this paper is to introduce a new sort of an interval valued fuzzy filters, called interval valued $(\alpha, \beta)$-fuzzy filters and to study interval valued $(\in, \in \vee q)_{\text {-fuzzy filters. To provide different }}$ characterizations of filters of an ordered semigroups in terms of an interval valued $(\in, \in \vee q)$-fuzzy filters. To extend our study of interval valued $(\in, \in \vee q)_{\text {-fuzzy left (right) and to investigate different }}$ characterizations of left (right)-filters of an ordered semigroups in terms of an interval valued $(\in, \in \vee q)$-fuzzy left (right) filters. Finally, we introduce the concepts of an interval valued fuzzy left (right) filters with thresholds.

\section{Preliminaries}

By an ordered semigroup (po-semigroup) we mean a structure $(S,,, \leq)$ in which the following properties are satisfied:

(OS1) $(S, \cdot)$ is a semigroup,

(OS2) $(S, \leq)$ is a poset,

(OS3) $a \leq b \rightarrow a x \leq b x$ and $x a \leq x b$ for all $a, b, x \in S$.

For a non-empty subset $A \subseteq S$, we denote ( $A]:=\{t \in S \mid t \leq h$ for some $h \in A\}$. If $A=\{a\}$, then we write ( $a]$ instead of $(\{a\}]$. For non-empty subsets $A, B \subseteq S$, we denote, $A B:=\{a b \mid a \in A, b \in B\}$.

Let $(S, ; \leq)$ be an ordered semigroup. A nonempty subset $A$ of $S$ is called a subsemigroup of $S$ if $A^{2} \subseteq A$.

Definition 2.1. A non-empty subset $F$ of an ordered semigroup $S$ is called a filter of $S$ if it satisfies the following conditions:

(b) $(\forall b \in S)(\forall a \in F) \quad a \leq b \rightarrow b \in F$,

(b) $(\forall a, b \in S) \quad a, b \in F \rightarrow a b \in F$,

(b) $(\forall a, b \in S) \quad a b \in F \rightarrow a, b \in F$.

$F$ is called a left (right) filter of $S$ if it satisfies conditions $\left(\mathrm{b}_{1}\right)$, $\left(b_{2}\right)$ of Definition 2.1, and

(b4) $(\forall a, b \in S) \quad a b \in F \rightarrow a \in F(b \in F)$.

Now, we recall some interval valued fuzzy logic concepts.

The interval valued fuzzy subsets provide a more adequate description of uncertainty than the traditional fuzzy subsets; it is therefore important to use interval valued fuzzy subsets in applications. In many practical applications, especially in fuzzy decision-making problems, the membership functions of objects are very individual, which are dependent on evaluation of experts in general and thus cannot be lightly confirmed. For example, concerning the fuzzy concept capability, there are three experts who give their evaluations to that of someone as $0.5,0.56$, and 0.6 , respectively. Clearly, it is more practical and reasonable to evaluate someone's capability by an interval valued data $[0.5,0.6]$ than a certain single value. By an interval number $\widetilde{a}$ we mean an interval $\left[a^{-}, a^{+}\right]$where $0 \leq a^{-} \leq a^{+} \leq 1$. The set of all interval numbers is denoted by $D[0,1]$. The interval $[a, a]$ can be simply identified by the number $a \in[0,1]$. For the interval numbers $\widetilde{a}_{i}=\left[a_{i}^{-}, a_{i}^{+}\right], \widetilde{b}_{i}=\left[b_{i}^{-}, b_{i}^{+}\right] \in D[0,0], i \in I$, we define;

$(\forall i \in I) r \max \left\{\widetilde{a}_{i}, \tilde{b}_{i}\right\}=\left[\max \left(a_{i}^{-}, b_{i}^{-}\right), \max \left(a_{i}^{+}, b_{i}^{+}\right)\right]$,

$(\forall i \in I) \quad r \min \left\{\widetilde{a}_{i}, \widetilde{b}_{i}\right\}=\left[\min \left(a_{i}^{-}, b_{i}^{-}\right), \min \left(a_{i}^{+}, b_{i}^{+}\right)\right]$,

$r \inf \widetilde{a}_{i}=\left[\hat{i \in I}_{i} a_{i \in I}^{-}, a_{i}^{+}\right), r \sup \widetilde{a}_{i}=\left[\underset{i \in I}{\vee} a_{i}^{-}, \underset{i \in I}{\vee} a_{i}^{+}\right)$,

$$
\begin{aligned}
& \widetilde{a}_{i}+\widetilde{b}_{i}=\left[a_{i}^{-}+b_{i}^{-}, a_{i}^{+}+b_{i}^{+}\right], \\
& \widetilde{a}_{i}-\widetilde{b}_{i}=\left\{\begin{array}{lll}
{\left[a_{i}^{-}-b_{i}^{-}, a_{i}^{+}-b_{i}^{+}\right], \quad \text { if }} & a_{i}^{-}+b_{i}^{-}<a_{i}^{+}+b_{i}^{+}, \\
{\left[a_{i}^{+}+b_{i}^{+}, a_{i}^{-}+b_{i}^{-}\right], \text {if }} & a_{i}^{-}+b_{i}^{-} \geq a_{i}^{+}+b_{i}
\end{array}\right. \\
& \widetilde{a}_{1} \leq \widetilde{a}_{2} \Leftrightarrow a_{1}^{-} \leq a_{2}^{-} \text {and } a_{1}^{+} \leq a_{2}^{+}, \\
& \widetilde{a}_{1}=\widetilde{a}_{2} \Leftrightarrow a_{1}^{-}=a_{2}^{-} \text {and } a_{1}^{+}=a_{2}^{+}, \\
& \tilde{a}_{1}<\widetilde{a}_{2} \Leftrightarrow \widetilde{a}_{1} \leq \widetilde{a}_{2} \text { and } \widetilde{a}_{1} \neq \widetilde{a}_{2}, \\
& k \widetilde{a}_{i}=\left[k a_{i}^{-}, k a_{i}^{+}\right], \text {whenever } 0 \leq k \leq 1 .
\end{aligned}
$$

Then, it is clear that $(D[0,1], \leq, \vee, \wedge)$ forms a complete lattice with $0=[0,0]$ as its least element and $1=[1,1]$ as its greatest element.

One of the main applications of fuzzy subsets is fuzzy control, and one of the most computationally intensive parts of fuzzy control is the defuzzification. Since a transition to interval valued fuzzy subsets usually increase the amount of computations, it is vitally important to design faster algorithms for the corresponding defuzzification.

An interval valued fuzzy subset $\widetilde{\mu}: X \rightarrow D[0,1]$ of $X$ is the set $\tilde{\mu}=\left\{x \in X \mid\left(x,\left[\mu^{-}(x), \mu^{+}(x)\right]\right) \in D[0,1]\right\}$, where $\mu^{-}$and $\mu^{+}$are two fuzzy subset such that $\mu^{-}(x) \leq \mu^{+}(x)$ for all $x \in X$. Let $\tilde{\mu}$ be an interval valued fuzzy subset of $X$. Then for every $[0,0] \leq \tilde{t} \leq[1,1]$ , the crisp set $\widetilde{\mu}_{\tilde{t}}=\{x \in X \mid \widetilde{\mu}(x) \geq \widetilde{t}\}$ is called the level set of $\tilde{\mu}$. We refer the reader to (18) for more details on operations on two interval-valued fuzzy sets of $X$.

Note that since every $a \in[0,1]$ is in correspondence with the interval $[a, a] \in D[0,1]$, hence a fuzzy set is a particular case of the interval valued fuzzy sets.

For any $\tilde{\mu}=\left[\mu^{-}, \mu^{+}\right]$and $\tilde{t}=\left[t^{-}, t^{+}\right], \quad$ we define $\widetilde{\mu}(x)+\widetilde{t}=\left[\mu^{-}(x)+t^{-}, \mu^{+}(x)+t^{+}\right]$for all $x \in X$. In particular, if $\mu^{-}(x)+t^{-}>1$ and $\mu^{+}(x)+t^{+}>1$, we write $\tilde{\mu}(x)+\widetilde{t}>[1,1]$.

Definition 2.2. An interval valued fuzzy subset $\tilde{\mu}$ of an ordered semigroup $(S, \cdot, \leq)$ is called an interval valued fuzzy filter of $S$ if it satisfies the following conditions:

$\left(\mathrm{b}_{5}\right)(\forall x, y \in S) \quad x \leq y \rightarrow \widetilde{\mu}(x) \leq \widetilde{\mu}(y)$,

(b) $(\forall x, y \in S) \quad \widetilde{\mu}(x y) \geq r \min \{\widetilde{\mu}(x), \widetilde{\mu}(y)\}$,

(b) $(\forall x, y \in S) \quad r \min \{\widetilde{\mu}(x), \widetilde{\mu}(y)\} \geq \widetilde{\mu}(x y)$.

$\widetilde{\mu}$ is called an interval valued fuzzy left (right) filter of $S$ if it satisfies condition $\left(\mathrm{b}_{5}\right)$ of Definition 2.2 and the following condition:

$\left(\mathrm{b}_{8}\right)(\forall x, y \in S) \quad \widetilde{\mu}(x y) \geq \widetilde{\mu}(x)(\widetilde{\mu}(x y) \geq \widetilde{\mu}(y))$.

The proof of the following theorem directly follows from the definition so is omitted.

Theorem 2.3. An interval valued fuzzy subset $\widetilde{\mu}$ of $S$ is an interval valued fuzzy filter of $S$ if and only if each non-empty level subset $\widetilde{\mu}_{\tilde{t}}$, for all $\widetilde{t} \in D(0,1]$ is a filter of $S$, respectively.

Example 2.4. Let $S=\{a, b, c, d, e, f\}$ be a set with the following order relation " $\leq$ " and multiplication as given in Table (1) and:

$\leq:=\{(a, a),(b, b),(c, c),(d, d),(e, e),(f, f),(a, d),(a, e),(d, e),(b, f),(b, e),(c, f),(c, e),(f, e)\}$ Table: 1 


\begin{tabular}{|l|l|l|l|l|l|l|}
\hline$\cdot$ & $a$ & $b$ & $c$ & $d$ & $e$ & $f$ \\
\hline$a$ & $a$ & $b$ & $b$ & $d$ & $e$ & $f$ \\
\hline$b$ & $b$ & $b$ & $b$ & $b$ & $b$ & $b$ \\
\hline$c$ & $b$ & $b$ & $b$ & $b$ & $b$ & $b$ \\
\hline$d$ & $d$ & $b$ & $b$ & $d$ & $e$ & $f$ \\
\hline$e$ & $e$ & $f$ & $f$ & $e$ & $e$ & $f$ \\
\hline$f$ & $f$ & $f$ & $f$ & $f$ & $f$ & $f$ \\
\hline
\end{tabular}

Then $(S, \dot{ }, \leq)$ is an ordered semigroup (see (17]). Here the filters of $S$ are $\{a, d, e\}$ and $S$. Define an interval valued fuzzy subset $\tilde{\mu}: S \rightarrow[0,1]$ by

$$
\tilde{\mu}(x)=\left(\begin{array}{ccccc}
a & b & c & d & e \\
{[0.6,0.66]} & {[0.4,0.5]} & {[0.3,0.4]} & {[0.7,0.76]} & {[0.8,0 . i}
\end{array}\right.
$$

then

$$
\tilde{\mu}(x)=\left\{\begin{array}{ccc}
S & \text { if } & {[0,0]<\tilde{t} \leq[0.3,0.4]} \\
\{a, d, e\} & \text { if } & {[0.5,0.5]<\tilde{t} \leq[0.6,0.66]} \\
\phi & \text { if } & {[08,0.8]<\tilde{t} \leq[1,1]}
\end{array}\right.
$$

Then $\tilde{\mu}$ is an interval valued fuzzy filter of $S$ for all $\tilde{t} \in D(0,1]$

An interval valued fuzzy subset $\tilde{\mu}$ of an ordered semigroup $(S, \cdot, \leq)$ of the form

$$
\widetilde{\mu}(y)=\left\{\begin{array}{ccc}
\tilde{t}(\neq[0,0]), & \text { if } & y=x, \\
{[0,0],} & \text { if } & y \neq x,
\end{array}\right.
$$

is called an interval valued fuzzy point with support $x$ and interval value $\tilde{t} \in D[0,1]$ and is denoted by $x_{\tilde{t}}$. For an interval valued fuzzy subset $\tilde{\mu}$ of $S$, we say that an interval valued fuzzy point $x_{\tilde{t}}$ is

(b9) contained in $\tilde{\mu}$, denoted by $x_{\tilde{t}} \in \tilde{\mu}$, if $\tilde{\mu}(x) \geq \tilde{t}$.

(b $\left.\mathrm{b}_{10}\right)$ quasi-coincident with $\tilde{\mu}$, denoted by $x_{\tilde{t}} q \tilde{\mu}$, if $\widetilde{\mu}(x)+\widetilde{t}>[1,1]$.

$\left(\mathrm{b}_{11}\right) \quad x_{\tilde{t}} \in \vee q \tilde{\mu}$, if $x_{\tilde{t}} \in \tilde{\mu}$ or $x_{\tilde{t}} q \tilde{\mu}$.

$\left(\mathrm{b}_{12}\right) \quad x_{\tilde{t}} \bar{\alpha} \tilde{\mu}$ if $x_{\tilde{t}} \alpha \tilde{\mu}$ does not hold for $\alpha \in\{\in, \mathrm{q}, \in \vee \mathrm{q}, \in \wedge \mathrm{q}\}$.

Let $S$ be an ordered semigroup and $\phi \neq A \subseteq S$. Then an interval valued characteristic function $\tilde{\chi}_{A}: S \rightarrow D[0,1]$ of $A$ is define by

$$
\tilde{\chi}_{A}(x)=\left\{\begin{array}{lll}
{[1,1],} & \text { if } & x \in A, \\
{[0,0],} & \text { if } & x \notin A .
\end{array}\right.
$$

Clearly, a non-empty subset $A$ of $S$ is a filter if and only if $\tilde{\chi}_{A}$ is an interval valued fuzzy filter of $S$.

\section{Interval valued ( $\alpha, \beta)$-fuzzy filters}

In what follows let $S$ denote an ordered semigroup, where, $\alpha$, $\beta$ denote any one of $\in, \mathrm{q}, \in \vee \mathrm{q}$, or $\in \wedge \mathrm{q}$ unless otherwise specified.

Theorem 3.1. Let $\tilde{\mu}$ be an interval valued fuzzy subset of $S$. Then $\tilde{\mu}_{\tilde{t}}$ is an interval valued filter of $S$ for all $\tilde{t} \in D(0.5,1]$ if and only if $\tilde{\mu}$ satisfies the following conditions:

(c $\left.c_{1}\right)(\forall x, y \in S) r \max \{\widetilde{\mu}(y),[0.5,0.5]\} \geq \widetilde{\mu}(x)$ with $x \leq y$, $\left(\mathrm{c}_{2}\right)(\forall x, y \in S) r \max \{\widetilde{\mu}(x y),[0.5,0.5]\} \geq r \min \{\widetilde{\mu}(x), \widetilde{\mu}(y)\}$

(c 3$)(\forall x, y \in S) r \max \{\tilde{\mu}(x), \tilde{\mu}(y),[0.5,0.5]\} \geq \tilde{\mu}(x y)$.

Proof. Assume that $\tilde{\mu}_{\tilde{t}} \neq \phi$ is an interval valued filter of $S$ for all $\tilde{t} \in D(0.5,1]$. If there exist $x, y \in S$ with $x \leq y$ such that the condition $\left(\mathrm{c}_{1}\right)$ is not valid, that is, there exist $(x, y \in S, x \leq y) \operatorname{rmax}\{\tilde{\mu}(y),[0.5,0.5]\}<\tilde{\mu}(y)=\tilde{r}$.

Then $\tilde{r} \in D(0.5,1], x \in \widetilde{\mu}_{\widetilde{r}}$ but $y \bar{\epsilon} \widetilde{\mu}_{\tilde{r}}$, a contradiction. Hence condition (c1) is valid. If there exist $x, y \in S$ such that $r \max \{\tilde{\mu}(x y),[0.5,0.5]\}<r \min \{\widetilde{\mu}(x), \widetilde{\mu}(y)\}=\widetilde{s} . \quad$ Then $\widetilde{s} \in D(0.5,1]$ and $x, y \in \widetilde{\mu}_{\widetilde{s}}$ but $x y \bar{\epsilon} \widetilde{\mu}_{\widetilde{s}}$, a contradiction. Hence condition $\left(\mathrm{c}_{2}\right)$ is valid. Also if there exist $x, y \in S$ such that $r \max \{\tilde{\mu}(x), \tilde{\mu}(y),[0.5,0.5]\}<\tilde{\mu}(x y)=\tilde{t}$. Then $\tilde{t} \in D(0.5,1]$, $x y \in \tilde{\mu}_{\tilde{t}}$ but $x \bar{\epsilon} \tilde{\mu}_{\tilde{t}}, y \bar{\epsilon} \tilde{\mu}_{\tilde{t}}$, again a contradiction. Hence condition $\left(c_{3}\right)$ is also valid.

Conversely, suppose that $\tilde{\mu}$ satisfies conditions $\left(c_{1}\right),\left(c_{2}\right)$ and $\left(c_{3}\right)$. Let $x, y \in S, x \leq y$ be such that $x \in \tilde{\mu}_{\tilde{t}}$ for some $\tilde{t} \in D(0.5,1]$, then $\quad \tilde{\mu}(x) \geq \tilde{t}$ and by condition $\quad\left(\mathrm{c}_{1}\right) \quad$ we have $r \max \{\tilde{\mu}(y),[0.5,0.5]\} \geq \tilde{\mu}(x) \geq \tilde{t}>[0.5,0.5]$. It follows that $\widetilde{\mu}(y) \geq \widetilde{t}$ that is $y \in \tilde{\mu}_{\tilde{t}}$.

For $\quad x, y \in \tilde{\mu}_{\tilde{t}} \quad$ and $\quad$ by $\quad\left(\mathrm{c}_{2}\right) \quad$ we get $r \max \{\tilde{\mu}(x y),[0.5,0.5]\} \geq r \min \{\tilde{\mu}(x), \tilde{\mu}(y)\} \geq \tilde{t}>[0.5,0.5]$, follows that $\tilde{\mu}(x y) \geq \tilde{t}$.

For $\quad x y \in \tilde{\mu}_{\tilde{t}}$, we have by $r \max \{\tilde{\mu}(x), \tilde{\mu}(y),[0.5,0.5]\} \geq \tilde{\mu}(x y) \geq \tilde{t}>[0.5,0.5]$ and hence $\tilde{\mu}(x) \geq \tilde{t}, \tilde{\mu}(y) \geq \tilde{t}$ it follows that $x, y \in \tilde{\mu}_{\tilde{t}}$. Thus $\tilde{\mu}_{\tilde{t}}$ is an interval valued filter of $S$ for all $\tilde{t} \in D(0.5,1]$.

The Conditions $\left(c_{1}\right)$ to $\left(c_{3}\right)$ of Definition 2.2, are equivalent to the following definition.

Definition 3.2. An interval valued fuzzy subset $\tilde{\mu}$ of an ordered semigroup $S$ is called an interval valued $(\in, \in)$-fuzzy filter of $S$ if it satisfies:

(c) $(\forall x, y \in S)(\forall \tilde{t} \in D(0,1]) x \leq y, x_{\tilde{t}} \in \tilde{\mu} \rightarrow y_{\tilde{t}} \in \tilde{\mu}$,

(c $\left.\mathrm{c}_{5}\right)(\forall x, y \in S)$

$(\forall \tilde{t}, \tilde{r} \in D(0,1])$ $x_{\tilde{t}} \in \tilde{\mu}, y_{\tilde{r}} \in \tilde{\mu} \rightarrow(x y)_{r \min \{\tilde{t}, \tilde{r}\}} \in \tilde{\mu}$,

(c) $(\forall x, y \in S)$

$(\forall \tilde{t} \in D(0,1])$ $(x y)_{\tilde{t}} \in \tilde{\mu} \rightarrow x_{\tilde{t}} \in \tilde{\mu}, y_{\tilde{t}} \in \tilde{\mu}$.

From Definition 3.3, we have the following theorem:

Theorem 3.3. An interval valued fuzzy subset $\tilde{\mu}$ of $S$ is an interval valued fuzzy filter of $S$ if and only if it satisfies the conditions $\left(c_{4}\right),\left(c_{5}\right)$ and $\left(c_{6}\right)$ of Definition 3.2.

Proof. It is straightforward.

Note that if $\widetilde{\mu}$ is an interval valued fuzzy subset $S$, defined by $\tilde{\mu}(x) \leq[0.5,0.5]$ for all $x \in S$, then the set, $\left\{x_{\tilde{t}} \mid x_{\tilde{t}} \in \wedge \mathrm{q} \tilde{\mu}\right\}$ is empty. Therefore, the case when $\alpha=\in \wedge \mathrm{q}$ is omitted in Definition 3.4 .

Definition 3.4. An interval valued fuzzy subset $\tilde{\mu}$ of $S$ is called an $(\alpha, \beta)$-fuzzy filter of $S$, where $\alpha \neq \in \wedge q$, if it satisfies the following conditions 
(c) $(\forall x, y \in S)(\forall \widetilde{t} \in D(0,1]) x \leq y, x_{\tilde{t}} \alpha \tilde{\mu} \rightarrow y_{\tilde{t}} \beta \widetilde{\mu}$,

(c) $(\forall x, y \in S)$

$(\forall \tilde{t}, \tilde{r} \in D(0,1])$

$x_{\tilde{t}} \alpha \tilde{\mu}, y_{\widetilde{r}} \alpha \tilde{\mu} \rightarrow(x y)_{r \min \{\tilde{\{}, \tilde{r}\}} \beta \tilde{\mu}$,

(c9) $(\forall x, y \in S)(\forall \tilde{t} \in D(0,1])(x y)_{\tilde{t}} \alpha \tilde{\mu} \rightarrow x_{\tilde{t}} \beta \tilde{\mu}, y_{\tilde{t}} \beta \tilde{\mu}$.

Theorem 3.5. Let $\tilde{\mu}$ be a non-zero interval valued $(\alpha, \beta)$-fuzzy filter of $S$. Then the set $\widetilde{\mu}_{[0,0]}:=\{x \in X \mid \widetilde{\mu}(x)>[0,0]\}$ is a filter of $S$.

Proof. If $x, y \in S, x \leq y$ and $x \in \widetilde{\mu}_{[0,0]}$, then $\widetilde{\mu}(x)>[0,0]$. Assume that $\widetilde{\mu}(y)=[0,0]$ and $\alpha \in\{\in, \in \vee \mathrm{q}\}$, then $x_{\widetilde{\mu}(x)} \alpha \widetilde{\mu}$ but $y_{\widetilde{\mu}(y)} \bar{\beta} \widetilde{\mu}$ for every $\beta \in\{\in, \mathrm{q} \in \vee \mathrm{q}, \in \wedge \mathrm{q}\}$ a contradiction. Note that $x_{[1,1]} \mathrm{q} \tilde{\mu} \quad$ but $\quad x_{[1,1]} \bar{\beta} \tilde{\mu} \quad$ for every $\quad \beta \in\{\in, \mathrm{q} \in \vee \mathrm{q}, \in \wedge q\} \quad$ a contradiction. Hence $\tilde{\mu}(y)>[0,0]$ that is $x \in \widetilde{\mu}_{[0,0]}$. Now if $x, y \in \widetilde{\mu}_{[0,0]}$, then $\tilde{\mu}(x)>[0,0]$ and $\tilde{\mu}(y)>[0,0]$. Assume that $\tilde{\mu}(x y)=[0,0]$ and let $\alpha \in\{\in, \in \vee \mathrm{q}\}$, then $x_{\widetilde{\mu}(x)} \alpha \tilde{\mu}$ and $y_{\widetilde{\mu}(y)} \alpha \tilde{\mu}$ but $(x y)_{r \min \{\widetilde{\mu}(x), \widetilde{\mu}(y)\}} \bar{\beta} \widetilde{\mu}$ for every $\beta \in\{\in \mathrm{q} \in \vee \mathrm{q}, \in \wedge \mathrm{q}\}$, a contradiction. Note that $x_{[1,1]} \mathrm{q} \tilde{\mu}$ and $y_{[1,1]} \mathrm{q} \tilde{\mu}$ but $(x y)_{r \min \{[1,1],[1,1]\}}=(x y)_{[1,1]} \bar{\beta} \widetilde{\mu}$ for every $\beta \in\{\in, \mathrm{q} \in \vee \mathrm{q}, \in \wedge \mathrm{q}\}$, a contradiction. Hence $\widetilde{\mu}(x y)>[0,0]$, it follows that $x y \in \widetilde{\mu}_{[0,0]}$. Let $x y \in \widetilde{\mu}_{[0,0]}$, then $\widetilde{\mu}(x y)>[0,0]$ and assume that $\widetilde{\mu}(x)=[0,0]$ or $\widetilde{\mu}(y)=[0,0]$ and $\alpha \in\{\in, \in \mathrm{vq}\}$, then $(x y)_{\tilde{\mu}(x y)} \alpha \tilde{\mu}$ but $(x)_{\widetilde{\mu}(x)} \bar{\beta} \widetilde{\mu}$ or $(y)_{\widetilde{\mu}(y)} \bar{\beta} \widetilde{\mu}$ for every $\beta \in\{\in, \mathrm{q} \in \vee \mathrm{q}, \in \wedge \mathrm{q}\}$, a contradiction. Note that $(x y)_{[1,1]} \mathrm{q} \widetilde{\mu}$ but $(x)_{[1,1]} \bar{\beta} \widetilde{\mu}$ or $(y)_{[1,1]} \bar{\beta} \widetilde{\mu}$ for every $\beta \in\{\in, \mathrm{q} \in \vee \mathrm{q}, \in \wedge \mathrm{q}\}$, a contradiction. Hence $\tilde{\mu}(x)>[0,0]$ and $\tilde{\mu}(x)>[0,0]$ so $x \in \widetilde{\mu}_{[0,0]}$ and $y \in \tilde{\mu}_{[0,0]}$. Therefore, $\widetilde{\mu}_{[0,0]}$ is a filter of $S$.

\section{Interval valued $(\in, \in \vee q)_{\text {-fuzzy filters }}$}

Now, we introduce the concept of an interval valued $(\in, \in \vee q)$ fuzzy filters in ordered semigroups and characterize filters of ordered semigroups in terms of interval valued $(\in, \in \vee q)$-fuzzy filters.

Definition 4.1. An interval valued fuzzy subset $\widetilde{\mu}$ of $S$ is called an interval valued $(\in, \in \vee \mathrm{q})$-fuzzy filter of $S$ if it satisfies the following conditions:

$\left(\mathrm{d}_{1}\right)(\forall x, y \in S) \quad(\forall \tilde{t} \in D(0,1]) x \leq y, x_{\tilde{t}} \in \tilde{\mu} \rightarrow y_{\tilde{t}} \in \vee \mathrm{q} \tilde{\mu}$,

$\left(\mathrm{d}_{2}\right)(\forall x, y \in S)$

$x_{\tilde{t}} \in \tilde{\mu}, y_{\widetilde{r}} \in \tilde{\mu} \rightarrow(x y)_{r \min \{\tilde{t}, \tilde{r}\}} \in \vee \mathrm{q} \tilde{\mu}$,

(d $\left.\mathrm{d}_{3}\right)(\forall x, y \in S)$

$(\forall \tilde{t}, \tilde{r} \in D(0,1])$

$(x y)_{\tilde{t}} \in \tilde{\mu} \rightarrow x_{\tilde{t}} \in \vee \mathrm{q} \tilde{\mu}, y_{\tilde{t}} \in \vee \mathrm{q} \tilde{\mu}$.

Example 4.2. Consider the ordered semigroup as given in Example 2.4, and define an interval valued fuzzy subset $\widetilde{\mu}$ by

$\widetilde{\mu}(x)=\left(\begin{array}{ccccc}a & b & c & d & e \\ {[0.6,0.7]} & {[0.3,0.4]} & {[0.5,0.6]} & {[0.7,0.8]} & {[0.8,0.9]}\end{array}\right.$

Then $\widetilde{\mu}$ is an interval valued $(\in, \in \vee \mathrm{q})$-fuzzy filter of $S$. But (i) $\tilde{\mu}$ is not an interval valued $(\in, \in)$-fuzzy filter of $S$, since $d_{[0.68,0.78]} \in \tilde{\mu} \quad$ and $\quad c_{[0.38,0.48]} \in \tilde{\mu}$

but

(dc) ${ }_{r \min \{[0.68,0.78],[0.38,0.48]\}}=b_{[0.38,0.48]} \bar{\epsilon} \tilde{\mu}$.

(ii) $\tilde{\mu}$ is not an interval valued (q, $\in$ )-fuzzy filter of $S$, since $d_{[0.78,0.88]} \mathrm{q} \tilde{\mu} \quad$ and $\quad f_{[0.88,0.89]} \mathrm{q} \tilde{\mu}$

$(d f)_{r \min \{[0.78,0.88],[0.88,0.89]\}}=f_{[0.78,0.88]} \bar{\epsilon} \tilde{\mu}$.

(iii) $\tilde{\mu}$ is not an interval valued $(\in, \mathrm{q})$-fuzzy filter of $S$, since $a_{[0.58,0.68]} \in \widetilde{\mu} \quad$ and $\quad f_{[0.38,0.448]} \in \widetilde{\mu} \quad$ but

$(\text { af })_{r \min \{[0.58,0.68],[0.38,0.48]\}}=f_{[0.38,0.48]} \overline{\mathrm{q}} \tilde{\mu}$.

Theorem 4.3. An interval valued fuzzy subset $\tilde{\mu}$ of $S$ is an interval valued $(\in, \in \vee q)$-fuzzy filter of $S$ if and only if it satisfies the following conditions:

$\left(\mathrm{d}_{4}\right)(\forall x, y \in S) \quad \widetilde{\mu}(y) \geq r \min \{\widetilde{\mu}(x),[0.5,0.5]\}$ with $x \leq y$,

(d) $(\forall x, y \in S) \tilde{\mu}(x y) \geq r \min \{\tilde{\mu}(x), \tilde{\mu}(y),[0.5,0.5]\}$,

(d $\left.\mathrm{d}_{6}\right)(\forall x, y \in S) r \min \{\widetilde{\mu}(x), \widetilde{\mu}(y)\} \geq r \min \{\widetilde{\mu}(x y),[0.5,0.5]\}$.

Proof. Let $\widetilde{\mu}$ be an $(\in, \in \vee \mathrm{q})$-fuzzy filter and $x, y \in S, x \leq y$. If $\tilde{\mu}(x)=[0,0], \quad$ then $\tilde{\mu}(y) \geq r \min \{\widetilde{\mu}(x),[0.5,0.5]\}$. Let $\widetilde{\mu}(x) \neq[0,0]$ and assume on contrary that $\tilde{\mu}(y)<r \min \{\tilde{\mu}(x),[0.5,0.5]\}$. Choose $\tilde{t} \in D(0,1]$ such that $\tilde{\mu}(y)<\tilde{t} \leq r \min \{\tilde{\mu}(x),[0.5,0.5]\}$. If $\tilde{\mu}(x)<[0.5,0.5]$, then $\tilde{\mu}(y)<\tilde{t} \leq \tilde{\mu}(x)$ and so $x_{\tilde{t}} \in \tilde{\mu}$ but $y_{\tilde{t}} \bar{\epsilon} \tilde{\mu}$ and $y_{\tilde{t}} \overline{\mathrm{q}} \tilde{\mu}$, a contradiction. If $\tilde{\mu}(x) \geq[0.5,0.5]$ then $\tilde{\mu}(y)<[0.5,0.5]$ and so $x_{[0.5,0.5]} \in \tilde{\mu}$ but $y_{[0.5,0.5]} \bar{\epsilon} \tilde{\mu}$ and $y_{[0.5,0.5]} \overline{\mathrm{q}} \tilde{\mu}$, again a contradiction. Hence $\tilde{\mu}(y) \geq r \min \{\widetilde{\mu}(x),[0.5,0.5]\}$ for all $x, y$ with $x \leq y$. Let $x, y \in S$ and if $\widetilde{\mu}(x)=[0,0]$ or $\widetilde{\mu}(y)=[0,0]$, then $\widetilde{\mu}(x y) \geq r \min \{\widetilde{\mu}(x), \widetilde{\mu}(y),[0.5,0.5]\}$. Let $\quad \tilde{\mu}(x) \neq[0,0]$, $\widetilde{\mu}(y) \neq[0,0]$ and assume on contrary that $\widetilde{\mu}(x y)<r \min \{\tilde{\mu}(x), \tilde{\mu}(y),[0.5,0.5]\}$. Choose $\tilde{s} \in D(0,1]$ such that $\quad \tilde{\mu}(x y)<\widetilde{s} \leq r \min \{\tilde{\mu}(x), \tilde{\mu}(y),[0.5,0.5]\}, \quad$ if $r \min \{\tilde{\mu}(x), \widetilde{\mu}(y)\}<[0.5,0.5], \quad$ then $\tilde{\mu}(x y)<\widetilde{s} \leq r \min \{\tilde{\mu}(x), \tilde{\mu}(y)\}$ follows that $x_{\widetilde{s}}, y_{\widetilde{s}} \in \tilde{\mu}$ but $(x y)_{\tilde{s}} \bar{\in} \tilde{\mu} \quad$ and $\quad(x y)_{\tilde{s}} \overline{\mathrm{q}} \tilde{\mu}, \quad$ a $\quad$ contradiction. Let $r \min \{\widetilde{\mu}(x), \widetilde{\mu}(y)\} \geq[0.5,0.5]$, then $\widetilde{\mu}(x y)<[0.5,0.5]$. It follows that $\quad x_{[0.5,0.5]} \in \tilde{\mu}, \quad y_{[0.5,0.5]} \in \tilde{\mu} \quad$ but $\quad(x y)_{[0.5,0.5]} \bar{\epsilon} \tilde{\mu} \quad$ and $(x y)_{[0.5,0.5]} \overline{\mathrm{q}} \tilde{\mu}, \quad$ again a contradiction. Hence $\widetilde{\mu}(x y) \geq r \min \{\widetilde{\mu}(x), \widetilde{\mu}(y),[0.5,0.5]\} \quad$ for all $\quad x, y \in S$. For $x, y \in S, \quad$ if $\quad \tilde{\mu}(x y)=[0,0], \quad$ then $r \min \{\widetilde{\mu}(x), \widetilde{\mu}(y)\} \geq r \min \{\widetilde{\mu}(x y),[0.5,0.5]\}$. Let $\widetilde{\mu}(x y) \neq[0,0]$ and assume on contrary that $r \min \{\widetilde{\mu}(x), \tilde{\mu}(y)\}<r \min \{\widetilde{\mu}(x y),[0.5,0.5]\} . \quad$ Choose $\tilde{r} \in D(0,1] \quad$ such that $r \min \{\widetilde{\mu}(x), \widetilde{\mu}(y)\}<\widetilde{r} \leq r \min \{\widetilde{\mu}(x y),[0.5,0.5]\} . \quad$ If $\widetilde{\mu}(x y)<[0.5,0.5]$, then $r \min \{\tilde{\mu}(x), \widetilde{\mu}(y)\}<\widetilde{r} \leq \widetilde{\mu}(x y)$ and so $(x y)_{\tilde{r}} \in \tilde{\mu}$ but $x_{\tilde{r}} \bar{\in} \tilde{\mu}$ and $y_{\widetilde{r}} \bar{\in} \tilde{\mu}$, a contradiction. If $\tilde{\mu}(x y) \geq[0.5,0.5]$ then $r \min \{\tilde{\mu}(x), \tilde{\mu}(y)\}<[0.5,0.5]$ and so $(x y)_{[0.5,0.5]} \in \tilde{\mu}$ but $x_{[0.5,0.5]} \bar{\epsilon} \tilde{\mu}, x_{[0.5,0.5]} \overline{\mathrm{q}} \widetilde{\mu}$ and $y_{[0.5,0.5]} \bar{\epsilon} \tilde{\mu}$, 
$y_{[0.5,0.5]} \overline{\mathrm{q}} \tilde{\mu}, \quad$ again $\quad$ a $\quad$ contradiction. Hence $r \min \{\tilde{\mu}(x), \tilde{\mu}(y)\} \geq r \min \{\tilde{\mu}(x y),[0.5,0.5]\}$ for all $x, y \in S$.

Conversely, let $x, y \in S, \quad x \leq y$ and $x_{\tilde{t}} \in \tilde{\mu}$ for some $\tilde{t} \in D(0,1]$, then $\tilde{\mu}(x) \geq \tilde{t}$. By hypothesis we have;

$$
\begin{aligned}
\tilde{\mu}(y) & \geq r \min \{\tilde{\mu}(x),[0.5,0.5]\} \\
& \geq r \min \{\tilde{t},[0.5,0.5]\} \\
& =\left\{\begin{array}{cll}
\tilde{t}, & \text { if } \tilde{t} \leq[0.5,0.5], \\
{[0.5,0.5],} & \text { if } \tilde{t}>[0.5,0.5],
\end{array}\right.
\end{aligned}
$$

It follows that $\tilde{\mu}(y) \geq \tilde{t}$ or $\tilde{\mu}(y)+\tilde{t}>[1,1]$, i.e., $x_{\tilde{t}} \in \vee \mathrm{q} \tilde{\mu}$. Let $x, y \in S$ and $\tilde{t}, \tilde{r} \in D(0,1]$ be such that $x_{\tilde{t}}, y_{\tilde{r}} \in \tilde{\mu}$. Then $\tilde{\mu}(x) \geq \tilde{t}, \quad \tilde{\mu}(y) \geq \tilde{r} \quad$ and $\quad$ so by $\left(\mathrm{d}_{5}\right)$ $\tilde{\mu}(x y) \geq r \min \{\tilde{\mu}(x), \tilde{\mu}(y),[0.5,0.5]\} \geq r \min \{\tilde{t}, \tilde{r},[0.5,0.5]\} . \quad$ If $r \min \{\tilde{t}, \tilde{r}\} \leq[0.5,0.5]$, then $\tilde{\mu}(x y) \geq r \min \{\tilde{t}, \tilde{r}\}$ follows that $(x y)_{r \min \{\tilde{t}, \tilde{r}\}} \in \tilde{\mu} . \quad$ If $\quad r \min \{\tilde{t}, \tilde{r}\}>[0.5,0.5], \quad$ then $\tilde{\mu}(x y)+r \min \{\tilde{t}, \tilde{r}\}>[0.5,0.5]+[0.5,0.5]=[1,1] \quad$ and $\quad$ so $(x y)_{r \min \{\tilde{t}, \tilde{r}\}} \mathrm{q} \tilde{\mu}$. Hence $(x y)_{r \min \{\tilde{t}, \tilde{r}\}} \in \vee \mathrm{q} \tilde{\mu}$. For $x, y \in S$, and $(x y)_{\tilde{t}} \in \tilde{\mu} \quad$ for $\quad$ some $\quad \tilde{t} \in D(0,1]$. Then by (d6) $r \min \{\tilde{\mu}(x), \tilde{\mu}(y)\} \geq r \min \{\tilde{\mu}(x y),[0.5,0.5]\} \geq r \min \{\tilde{t},[0.5,0.5]\}$ . If $\tilde{t} \leq[0.5,0.5]$ then $r \min \{\tilde{\mu}(x), \tilde{\mu}(y)\} \geq \widetilde{t}$ and so $x_{\tilde{t}}, y_{\tilde{t}} \in \tilde{\mu}$. If

$$
\tilde{t}>[0.5,0.5]
$$

then $r \min \{\tilde{\mu}(x), \tilde{\mu}(y)\}+\tilde{t}>[0.5,0.5]+[0.5,0.5]=[1,1] \quad$ that $\quad$ is, $\tilde{\mu}(x)+\tilde{t}>[1,1]$ and $\tilde{\mu}(y)+\tilde{t}>[1,1]$, follows that $x_{\tilde{t}} \mathrm{q} \tilde{\mu}$ and $y_{\tilde{t}} \mathrm{q} \tilde{\mu}$. Therefore $x_{\tilde{t}} \in \vee \mathrm{q} \tilde{\mu}$ and $y_{\tilde{t}} \in \vee \mathrm{q} \tilde{\mu}$. Consequently, $\tilde{\mu}$ is an interval valued $(\in, \in \vee q)$-fuzzy filter of $S$.

Remark 4.4. An interval valued fuzzy subset $\tilde{\mu}$ of an ordered semigroup $S$ is an interval valued $(\in, \in \vee \mathrm{q})$-fuzzy filter of $S$ if and only if it satisfies conditions $\left(\mathrm{d}_{4}\right),\left(\mathrm{d}_{5}\right)$ and $\left(\mathrm{d}_{6}\right)$ of Theorem 4.3 .

Remark 4.5. By Remark 4.4, every interval valued fuzzy filter of an ordered semigroup $S$ is an interval valued $(\in, \in \vee q)$-fuzzy filter of $S$. However, the converse is not true, in general.

Example 4.6. Consider the ordered semigroup as given in Example 2.4, and define an interval valued fuzzy subset $\tilde{\mu}$ as follows:

$$
\tilde{\mu}(x)=\left(\begin{array}{ccccc}
a & b & c & d & e \\
{[0.6,0.7]} & {[0.3,0.4]} & {[0.5,0.6]} & {[0.7,0.8]} & {[0.8,0.9]}
\end{array}\right.
$$

Then $\tilde{\mu}$ is an interval valued $(\in, \in \vee q)$-fuzzy filter of $S$. But $\tilde{\mu}$ is not an interval valued $(\alpha, \beta)$-fuzzy filter of $S$, where $\alpha \in\{\in, \mathrm{q}, \in \vee \mathrm{q}\}$ and $\beta \in\{\in, \mathrm{q},, \in \vee \mathrm{q}, \in \wedge \mathrm{q}\}$, as shown in Example 4.2.

Using Theorem 4.3, we have the following characterization of fuzzy filters of ordered semigroups.

Theorem 4.7. Let $(S, ;, \leq)$ be an ordered semigroup and $\phi \neq A \subseteq S$. Then $A$ is a filter of $S$ if and only if the characteristic function $\tilde{\chi}_{A}$ of $A$ is an interval valued $(\in, \in \vee \mathrm{q})$-fuzzy filter of $S$.

Theorem 4.8. Let $A$ be a filter of $S$ and $\widetilde{\mu}$ is an interval valued fuzzy subset of $S$ such that

$$
\tilde{\mu}(x):=\left\{\begin{array}{cl}
\geq[0.5,0.5], & \text { if } \quad x \in A, \\
{[0,0],} & \text { if } \quad x \in S \backslash A .
\end{array}\right.
$$

(a) $\tilde{\mu}$ is an interval valued $(\mathrm{q}, \in \vee \mathrm{q})$-fuzzy filter of $S$.

(b) $\tilde{\mu}$ is an interval valued $(\in, \in \vee \mathrm{q})$-fuzzy filter of $S$.

Proof (a). Let $x, y \in S, x \leq y$ and $\tilde{t} \in D(0,1]$ be such that $x_{\tilde{t}} \mathrm{q} \tilde{\mu}$. Then $x \in A$ and $y \geq x \in A$ implies $y \in A$. If $\tilde{t} \leq[0.5,0.5]$ then $\tilde{\mu}(y) \geq[0.5,0.5] \geq \tilde{t}$ and so $y_{\tilde{t}} \in \tilde{\mu}$. If $\tilde{t}>[0.5,0.5]$ then $\tilde{\mu}(y)+\tilde{t} \geq[0.5,0.5]+[0.5,0.5]=[1,1]$ and $y_{\tilde{t}} \mathrm{q} \tilde{\mu}$. Hence $y_{\tilde{t}} \in \vee \mathrm{q} \tilde{\mu}$. Let $x, y \in S$ and $\tilde{t}, \tilde{r} \in D(0,1]$ be such that $x_{\tilde{t}} \mathrm{q} \tilde{\mu}$ and $y_{\tilde{r}} \mathrm{q} \tilde{\mu}$. Then $x, y \in A$ and so $x y \in A$. If $r \min \{\tilde{t}, \tilde{r}\} \leq[0.5,0.5]$, then $\tilde{\mu}(x y) \geq[0.5,0.5] \geq r \min \{\tilde{t}, \tilde{r}\}$ and so $\quad(x y)_{r \min \{\tilde{t}, \tilde{r}\}} \in \tilde{\mu}$. If $\quad r \min \{\tilde{t}, \tilde{r}\}>[0.5,0.5]$ then $\tilde{\mu}(x y)+r \min \{\tilde{t}, \tilde{r}\}>[0.5,0.5]+[0.5,0.5]=[1,1] \quad$ and $\quad$ so $(x y)_{r \min \{\tilde{t}, \tilde{r}\}} \mathrm{q} \tilde{\mu}$. Hence $(x y)_{r \min \{\tilde{t}, \tilde{r}\}} \in \vee \mathrm{q} \tilde{\mu}$. For $x, y \in S$ and $(x y)_{\tilde{t}} \mathrm{q} \tilde{\mu}$ for some $\tilde{t} \in D(0,1]$ we have $x y \in A$ then $x, y \in A$. If $\tilde{t} \leq[0.5,0.5]$ then $r \min \{\tilde{\mu}(x), \tilde{\mu}(y)\} \geq[0.5,0.5] \geq \tilde{t}$ follows that $\tilde{\mu}(x) \geq \tilde{t}$ and $\tilde{\mu}(y) \geq \tilde{t}$. Hence $x_{\tilde{t}} \in \tilde{\mu}$ and $y_{\tilde{t}} \in \tilde{\mu}$. If $\tilde{t}>[0.5,0.5]$ then $r \min \{\tilde{\mu}(x), \tilde{\mu}(y)\}+\tilde{t}>[0.5,0.5]+[0.5,0.5]=[1,1] \quad$ that is $\tilde{\mu}(x)+\tilde{t}>[1,1]$ and $\tilde{\mu}(y)+\tilde{t}>[1,1]$, it follows that $x_{\tilde{t}} \mathrm{q} \tilde{\mu}$ and $y_{\tilde{t}} \mathrm{q} \tilde{\mu}$. Hence $x_{\tilde{t}} \in \vee \mathrm{q} \tilde{\mu}$ and $y_{\tilde{t}} \in \vee \mathrm{q} \tilde{\mu}$.

(b) Let $x, y \in S, x \leq y$ and $\tilde{t} \in D(0,1]$ be such that $x_{\tilde{t}} \in \tilde{\mu}$. Then $x \in A$ and we have $y \in A$. If $\tilde{t} \leq[0.5,0.5]$ then $\tilde{\mu}(y) \geq[0.5,0.5] \geq \tilde{t}$ and so $y_{\tilde{t}} \in \tilde{\mu}$. If $\tilde{t}>[0.5,0.5]$ then $\tilde{\mu}(y)+\tilde{t} \geq[0.5,0.5]+[0.5,0.5]=[1,1]$ and $y_{\tilde{t}} \mathrm{q} \tilde{\mu}$. Hence, $y_{\tilde{t}} \in \vee \mathrm{q} \tilde{\mu}$. Let $x, y \in S$ and $\tilde{t}, \tilde{r} \in D(0,1]$ be such that $x_{\tilde{t}} \in \tilde{\mu}$ and $y_{\tilde{r}} \in \tilde{\mu}$. Then $x, y \in A$ and we have $x y \in A$. If $r \min \{\tilde{t}, \tilde{r}\} \leq[0.5,0.5] \quad$ then $\quad \tilde{\mu}(x y) \geq[0.5,0.5] \geq r \min \{\tilde{t}, \tilde{r}\}$ follows that $(x y)_{r \min \{\tilde{t}, \tilde{r}\}} \in \tilde{\mu}$. If $r \min \{\tilde{t}, \tilde{r}\}>[0.5,0.5]$ then $\tilde{\mu}(x y)+r \min \{\tilde{t}, \tilde{r}\}>[0.5,0.5]+[0.5,0.5]=[1,1] \quad$ and $\quad$ so $(x y)_{r \min \{\tilde{t}, \tilde{r}\}} \mathrm{q} \tilde{\mu}$. Hence $(x y)_{r \min \{\tilde{r}, \tilde{r}\}} \in \vee \mathrm{q} \tilde{\mu}$. For $x, y \in S$ and $(x y)_{\tilde{t}} \in \tilde{\mu}$ for some $\tilde{t} \in D(0,1]$ we have $x y \in A$ then $x, y \in A$. If $\tilde{t} \leq[0.5,0.5]$ then $r \min \{\tilde{\mu}(x), \tilde{\mu}(y)\} \geq[0.5,0.5] \geq \tilde{t}$ follows that $\tilde{\mu}(x) \geq \tilde{t}$ and $\tilde{\mu}(y) \geq \tilde{t}$. Hence $x_{\tilde{t}} \in \tilde{\mu}$ and $y_{\tilde{t}} \in \tilde{\mu}$. If $\tilde{t}>[0.5,0.5]$ then $r \min \{\tilde{\mu}(x), \tilde{\mu}(y)\}+\tilde{t}>[0.5,0.5]+[0.5,0.5]=[1,1] \quad$ that is $\tilde{\mu}(x)+\tilde{t}>[1,1]$ and $\tilde{\mu}(y)+\tilde{t}>[1,1]$, it follows that $x_{\tilde{t}} \mathrm{q} \tilde{\mu}$ and $y_{\tilde{t}} \mathrm{q} \tilde{\mu}$. Hence $x_{\tilde{t}} \in \vee \mathrm{q} \tilde{\mu}$ and $y_{\tilde{t}} \in \vee \mathrm{q} \tilde{\mu}$.

From Example 4.2, we see that an interval valued $(\in, \in \vee q)$-fuzzy filter of $S$ may not be an interval valued $(\mathrm{q}, \in \vee \mathrm{q})$-fuzzy filter of $S$ (see Example 4.2 (ii)).

In the following theorem we give a condition for an interval valued ( $\in, \in \vee q)$-fuzzy filter to be an interval valued $(\in, \in)$-fuzzy filter of $S$.

Theorem 4.9. Let $\tilde{\mu}$ be an interval valued $(\in, \in \vee q)$-fuzzy filter of $S$ such that $\tilde{\mu}(x)<[0.5,0.5]$ for all $x \in S$. Then $\tilde{\mu}$ is an interval valued $(\in, \in)$-fuzzy filter of $S$.

Then, 
Proof. Let $x, y \in S, x \leq y$ and $x_{\tilde{t}} \in \tilde{\mu}$ for some $\tilde{t} \in D(0,1]$. Then $\tilde{\mu}(x) \geq \widetilde{t}$ and by $\left(\mathrm{d}_{4}\right)$

$$
\begin{aligned}
\tilde{\mu}(y) & \geq r \min \{\tilde{\mu}(x),[0.5,0.5]\} \\
& \geq r \min \{\tilde{t},[0.5,0.5]\} \\
& =\widetilde{t} .
\end{aligned}
$$

Hence $y_{\tilde{t}} \in \tilde{\mu}$.

Let $x, y \in S$ and $\tilde{t}, \tilde{r} \in D(0,1]$ be such that $x_{\tilde{t}} \in \tilde{\mu}$ and $y_{\tilde{r}} \in \tilde{\mu}$. Then $\tilde{\mu}(x) \geq \tilde{t}$ and $\tilde{\mu}(y) \geq \widetilde{r}$ and so by $\left(\mathrm{d}_{5}\right)$

$\tilde{\mu}(x y) \geq r \min \{\tilde{\mu}(x), \tilde{\mu}(y),[0.5,0.5]\}$

$$
\begin{aligned}
& =r \min \{\tilde{\mu}(x), \tilde{\mu}(y)\} \\
& \geq r \min \{\tilde{t}, \tilde{r}\} .
\end{aligned}
$$

Hence $(x y)_{r \min \{\tilde{t}, \tilde{r}\}} \in \tilde{\mu}$.

Now, let $x, y \in S$ and $\tilde{t} \in D(0,1]$ be such that $(x y)_{\tilde{t}} \in \tilde{\mu}$. Then $\tilde{\mu}(x y) \geq \tilde{t}$ and so by $\left(\mathrm{d}_{6}\right)$

$r \min \{\tilde{\mu}(x), \tilde{\mu}(y)\} \geq r \min \{\tilde{\mu}(x y),[0.5,0.5]\}$

$$
\begin{aligned}
& =\tilde{\mu}(x y) \\
& \geq \tilde{t} .
\end{aligned}
$$

Hence, $x_{\tilde{t}} \in \tilde{\mu}$ and $y_{\tilde{t}} \in \tilde{\mu}$.

Theorem 4.10. An interval valued fuzzy subset $\tilde{\mu}$ of $S$ is an interval valued $(\in, \in \vee \mathrm{q})$-fuzzy filter of $S$ if and only if the set $\tilde{\mu}_{\tilde{t}}:=\{x \in X \mid \tilde{\mu}(x)>\widetilde{t}\} \quad$ is $\quad$ a filter of $\quad S \quad$ for all $[0,0]<\tilde{t} \leq[0.5,0.5]$.

Proof. Assume that $\tilde{\mu}$ is an interval valued $(\in, \in \vee q)$-fuzzy filter of $S$. Let $x, y \in S, x \leq y$ and let $[0,0]<\tilde{t} \leq[0.5,0.5]$ be such that $x \in \tilde{\mu}_{\tilde{t}}$. Then $\tilde{\mu}(x) \geq \tilde{t}$ and it follows from Theorem 4.3 $\left(\mathrm{d}_{4}\right)$ that

$$
\begin{aligned}
\widetilde{\mu}(y) & \geq r \min \{\tilde{\mu}(x),[0.5,0.5]\} \\
& \geq r \min \{\tilde{t},[0.5,0.5]\} \\
& =\widetilde{t},
\end{aligned}
$$

and so $y \in \tilde{\mu}_{\tilde{t}}$. Let $x, y \in \tilde{\mu}_{\tilde{t}}$ for some $[0,0]<\tilde{t} \leq[0.5,0.5]$. Then $\tilde{\mu}(x) \geq \tilde{t}$ and $\tilde{\mu}(y) \geq \tilde{t}$. From Theorem $4.3\left(\mathrm{~d}_{5}\right)$ it follows that $\tilde{\mu}(x y) \geq r \min \{\tilde{\mu}(x), \tilde{\mu}(y),[0.5,0.5]\}$

$$
\begin{aligned}
& \geq r \min \{\tilde{t},[0.5,0.5]\} \\
& =\widetilde{t},
\end{aligned}
$$

and so $\quad x y \in \tilde{\mu}_{\tilde{t}}$. Now, let $\quad x y \in \tilde{\mu}_{\tilde{t}}$ for some $[0,0]<\tilde{t} \leq[0.5,0.5]$. Then $\tilde{\mu}(x y) \geq \tilde{t}$ and so by $\left(\mathrm{d}_{6}\right)$, we have $r \min \{\tilde{\mu}(x), \tilde{\mu}(y)\} \geq r \min \{\tilde{\mu}(x y),[0.5,0.5]\}$

$$
\begin{aligned}
& \geq r \min \{\tilde{t},[0.5,0.5]\} \\
& \geq \tilde{t},
\end{aligned}
$$

i.e., $\tilde{\mu}(x) \geq \tilde{t}$ and $\tilde{\mu}(y) \geq \widetilde{t}$. It follows that $x, y \in \tilde{\mu}_{\tilde{t}}$.

Conversely, let for all $[0,0]<\tilde{t} \leq[0.5,0.5]$ the set $\tilde{\mu}_{\tilde{t}}:=\{x \in X \mid \tilde{\mu}(x)>\widetilde{t}\}$ is a filter of $S$. If there exist $x, y \in S$, $x \leq y$ such that $\tilde{\mu}(y)<r \min \{\tilde{\mu}(x),[0.5,0.5]\}$, then we can choose $[0,0]<\tilde{t} \leq[0.5,0.5]$ such that $\tilde{\mu}(y)<\tilde{t} \leq r \min \{\tilde{\mu}(x),[0.5,0.5]\}$ , then $x \in \tilde{\mu}_{\tilde{t}}$ but $y \bar{\epsilon} \tilde{\mu}_{\tilde{t}}$, a contradiction. Hence $\tilde{\mu}(y) \geq r \min \{\tilde{\mu}(x),[0.5,0.5]\}$ for all $x, y \in S$ with $x \leq y$. If there exist $x, y \in S$ such that $\tilde{\mu}(x y)<r \min \{\tilde{\mu}(x), \tilde{\mu}(y),[0.5,0.5]\}$. We can choose $[0,0]<\widetilde{s} \leq[0.5,0.5]$ such that $\tilde{\mu}(x y)<\tilde{s} \leq r \min \{\tilde{\mu}(x), \tilde{\mu}(y),[0.5,0.5]\}$. Then $x, y \in \tilde{\mu}_{\widetilde{s}}$ but $x y \bar{\epsilon} \tilde{\mu}_{\tilde{s}}, \quad$ a $\quad$ contradiction. Hence $\tilde{\mu}(x y) \geq r \min \{\tilde{\mu}(x), \tilde{\mu}(y),[0.5,0.5]\}$ for all $x, y \in S$. Finally, if there exist $\quad x, y \in S \quad$ such that $r \min \{\tilde{\mu}(x), \tilde{\mu}(y)\}<r \min \{\widetilde{\mu}(x y),[0.5,0.5]\} \quad$ then $r \min \{\tilde{\mu}(x), \tilde{\mu}(y)\}<\tilde{r} \leq r \min \{\tilde{\mu}(x y),[0.5,0.5]\} \quad$ for $\quad$ some $[0,0]<\widetilde{r} \leq[0.5,0.5]$. Thus $x y \in \widetilde{\mu}_{\widetilde{r}}$ but $x, y \bar{\in} \tilde{\mu}_{\tilde{r}}$. Since $\tilde{\mu}_{\widetilde{r}}$ is a filter of $S$, and $x y \in \widetilde{\mu}_{\widetilde{r}}$ then $x, y \in \widetilde{\mu}_{\widetilde{r}}$, a contradiction. Hence $r \min \{\tilde{\mu}(x), \tilde{\mu}(y)\} \geq r \min \{\tilde{\mu}(x y),[0.5,0.5]\}$ for all $x, y \in S$.

For any interval valued fuzzy subset $\tilde{\mu}$ of an ordered semigroup $S$ and $\tilde{t} \in D(0,1]$, we consider the following two subsets $Q(\tilde{\mu} ; \tilde{t}):=\left\{x \in S \mid x_{\tilde{t}} \mathrm{q} \tilde{\mu}\right\}$ and $[\tilde{\mu}]_{\tilde{t}}:=\left\{x \in S \mid x_{\tilde{t}} \in \vee \mathrm{q} \tilde{\mu}\right\}$.

It is clear that $[\tilde{\mu}]_{\tilde{t}}=\tilde{\mu}_{\tilde{t}} \cup Q(\tilde{\mu} ; \tilde{t})$.

Theorem 4.11. An interval valued fuzzy subset $\tilde{\mu}$ of $S$ is an interval valued $(\in, \in \vee \mathrm{q})$-fuzzy filter of $S$ if and only if $[\tilde{\mu}]_{\tilde{t}}(=\phi)$ is a filter of $S$ for all $[0,0]<\tilde{t} \leq[1,1]$.

Proof. Assume that $\tilde{\mu}$ is an interval valued $(\in, \in \vee q)$-fuzzy filter of $S$ and let $[0,0]<\tilde{t} \leq[1,1]$ be such that $[\tilde{\mu}]_{\tilde{t}}(\neq \phi)$. Let $x, y \in S, x \leq y$ and $x \in[\tilde{\mu}]_{\tilde{t}}$. Then $\tilde{\mu}(x) \geq \tilde{t}$ or $\tilde{\mu}(x)+\tilde{t}>[1,1]$ . Since $\tilde{\mu}$ is an interval valued $(\in, \in \vee q)$-fuzzy filter and $x \leq y$, we have by (d4) $\tilde{\mu}(y) \geq r \min \{\widetilde{\mu}(x),[0.5,0.5]\}$.

(i). If $\quad \tilde{\mu}(x) \geq \tilde{t} \quad$ and $\quad \tilde{t}>[0.5,0.5]$, then $\widetilde{\mu}(y) \geq r \min \{\widetilde{\mu}(x),[0.5,0.5]\} \geq r \min \{\tilde{t},[0.5,0.5]\}=[0.5,0.5]$ and so $\tilde{\mu}(y)+\widetilde{t}>[0.5,0.5]+[0.5,0.5]=[1,1]$. Hence $y_{\tilde{t}} \mathrm{q} \tilde{\mu}$ i.e., $y \in Q(\tilde{\mu} ; \tilde{t}) \subseteq[\tilde{\mu}]_{\tilde{t}} . \quad$ If $\quad \tilde{t} \leq[0.5,0.5] \quad$ then $\tilde{\mu}(y) \geq r \min \{\tilde{\mu}(x),[0.5,0.5]\} \geq r \min \{\tilde{t},[0.5,0.5]\}=\widetilde{t}$ and so $y_{\tilde{t}} \in \tilde{\mu}$ i.e., $y \in \tilde{\mu}_{\tilde{t}} \subseteq[\tilde{\mu}]_{\tilde{t}}$.

(ii). If $\tilde{\mu}(x)+\tilde{t}>[1,1]$ and $\tilde{t}>[0.5,0.5]$, then

$\tilde{\mu}(y) \geq r \min \{\tilde{\mu}(x),[0.5,0.5]\}$

$$
\begin{aligned}
& \geq r \min \{[1,1]-\tilde{t},[0.5,0.5]\} \\
& =[1,1]-\tilde{t},
\end{aligned}
$$

that is $\tilde{\mu}(y)+\tilde{t}>[1,1]$ and hence $y_{\tilde{t}} \mathrm{q} \tilde{\mu}$, i.e., $y \in Q(\tilde{\mu} ; \tilde{t}) \subseteq[\tilde{\mu}]_{\tilde{t}}$. If $\tilde{t} \leq[0.5,0.5]$, then

$$
\begin{aligned}
\tilde{\mu}(y) & \geq r \min \{\tilde{\mu}(x),[0.5,0.5]\} \\
& \geq r \min \{[1,1]-\tilde{t},[0.5,0.5]\} \\
& =[0.5,0.5] \geq \tilde{t},
\end{aligned}
$$

and so $y_{\tilde{t}} \in \tilde{\mu}$, hence $y \in \widetilde{\mu}_{\tilde{t}} \subseteq[\tilde{\mu}]_{\tilde{t}}$. Thus in both cases, we have $y \in[\tilde{\mu}]_{\tilde{t}}$.

If $x, y \in[\tilde{\mu}]_{\tilde{t}}$, then $\tilde{\mu}(x) \geq \tilde{t} \quad$ or $\quad \tilde{\mu}(x)+\tilde{t}>[1,1]$ and $\tilde{\mu}(y) \geq \tilde{t}$ or $\tilde{\mu}(y)+\widetilde{t}>[1,1]$. We consider the following four cases:

(i) $\tilde{\mu}(x) \geq \tilde{t}$ and $\tilde{\mu}(y) \geq \tilde{t}$,

(ii) $\tilde{\mu}(x) \geq \tilde{t}$ and $\tilde{\mu}(y)+\widetilde{t}>[1,1]$,

(iii) $\tilde{\mu}(x)+\tilde{t}>[1,1]$ and $\tilde{\mu}(y) \geq \tilde{t}$,

(iv) $\tilde{\mu}(x)+\tilde{t}>[1,1]$ and $\tilde{\mu}(y)+\tilde{t}>[1,1]$. 
Using Case (i) in Theorem $4.3\left(\mathrm{~d}_{5}\right)$ we have;

$\widetilde{\mu}(x y) \geq r \min \{\tilde{\mu}(x), \tilde{\mu}(y),[0.5,0.5]\}$

$$
\begin{aligned}
& \geq r \min \{\tilde{t},[0.5,0.5]\} \\
& =\left\{\begin{array}{cl}
{[0.5,0.5],} & \text { if } \tilde{t}>[0.5,0.5], \\
\tilde{t}, & \text { if } \quad \tilde{t} \leq[0.5,0.5],
\end{array}\right.
\end{aligned}
$$

and so $\tilde{\mu}(x y)+\widetilde{t}>[0.5,0.5]+[0.5,0.5]=[1,1]$ or $\tilde{\mu}(x y) \geq \tilde{t}$.

Therefore $x y \in \tilde{\mu}_{\tilde{t}} \cup Q(\tilde{\mu} ; \tilde{t})=[\tilde{\mu}]_{\tilde{t}}$.

For Case (ii), assume that $\tilde{t}>[0.5,0.5]$, then $[1,1]-\tilde{t}<[0.5,0.5]$. If $r \min \{\tilde{\mu}(y),[0.5,0.5]\} \leq \tilde{\mu}(x)$, then by (d5)

$\tilde{\mu}(x y) \geq r \min \{\tilde{\mu}(x), \tilde{\mu}(y),[0.5,0.5]\} \geq r \min \{\tilde{\mu}(y),[0.5,0.5]\}>[1$, , and if $r \min \{\tilde{\mu}(y),[0.5,0.5]\}>\widetilde{\mu}(x)$ then $\tilde{\mu}(x y) \geq \tilde{\mu}(x) \geq \tilde{t}$. Hence $\quad x y \in \tilde{\mu}_{\tilde{t}} \cup Q(\tilde{\mu} ; \tilde{t})=[\tilde{\mu}]_{\tilde{t}}$ for $\quad \tilde{t}>[0.5,0.5]$. If $\tilde{t} \leq[0.5,0.5], \quad$ then $\quad[1,1]-\tilde{t} \geq[0.5,0.5]$. If $r \min \{\tilde{\mu}(x),[0.5,0.5]\} \leq \widetilde{\mu}(y), \quad$ then then by $\left(\mathrm{d}_{5}\right)$ $\tilde{\mu}(x y) \geq r \min \{\tilde{\mu}(x), \tilde{\mu}(y),[0.5,0.5]\} \geq r \min \{\tilde{\mu}(x),[0.5,0.5]\} \geq \tilde{t}$ and $\quad$ if $\quad r \min \{\tilde{\mu}(x),[0.5,0.5]\}>\widetilde{\mu}(y), \quad$ then $\tilde{\mu}(x y) \geq \tilde{\mu}(y) \geq[1,1]-\tilde{t}$. Thus $x y \in \tilde{\mu}_{\tilde{t}} \cup Q(\tilde{\mu} ; \tilde{t})=[\tilde{\mu}]_{\tilde{t}}$ for $\tilde{t} \leq[0.5,0.5]$. For the case (iii), we have same discussion as in case (ii). For case (iv), if $\tilde{t}>[0.5,0.5]$ then $[1,1]-\tilde{t}<[0.5,0.5]$ and by $\left(\mathrm{d}_{5}\right)$ we have

$\tilde{\mu}(x y) \geq r \min \{\tilde{\mu}(x), \tilde{\mu}(y),[0.5,0.5]\}$

$$
=\left\{\begin{array}{cll}
{[0.5,0.5]>[1,1]-\tilde{t},} & \text { if } & r \min \{\tilde{\mu}(x), \tilde{\mu}(y) \\
r \min \{\tilde{\mu}(x), \widetilde{\mu}(y)\}>[1,1]-\tilde{t}, & \text { if } & r \min \{\tilde{\mu}(x), \tilde{\mu}(y)
\end{array}\right.
$$

and $\quad$ so $\quad x y \in Q(\tilde{\mu} ; \tilde{t}) \subseteq[\tilde{\mu}]_{\tilde{t}}$. If $\quad \tilde{t} \leq[0.5,0.5]$, then

$[1,1]-\tilde{t} \geq[0.5,0.5]$. Thus

$\tilde{\mu}(x y) \geq r \min \{\tilde{\mu}(x), \tilde{\mu}(y),[0.5,0.5]\}$

$$
=\left\{\begin{array}{cll}
{[0.5,0.5] \geq \tilde{t},} & \text { if } & r \min \{\widetilde{\mu}(x), \widetilde{\mu}(y) \\
r \min \{\tilde{\mu}(x), \widetilde{\mu}(y)\}>[1,1]-\widetilde{t}, & \text { if } & r \min \{\tilde{\mu}(x), \widetilde{\mu}(y)
\end{array}\right.
$$

which implies that $x y \in \tilde{\mu}_{\tilde{t}} \cup Q(\tilde{\mu} ; \tilde{t})=[\tilde{\mu}]_{\tilde{t}}$. Let $x y \in[\tilde{\mu}]_{\tilde{t}}$, then $\tilde{\mu}(x y) \geq \tilde{t}$ or $\tilde{\mu}(x y)+\tilde{t}>[1,1]$. Assume that $\tilde{\mu}(x y) \geq \tilde{t}$, then by $\left(\mathrm{d}_{6}\right)$

$r \min \{\tilde{\mu}(x), \tilde{\mu}(y)\} \geq r \min \{\tilde{\mu}(x y),[0.5,0.5]\}$

$$
\begin{aligned}
& \geq r \min \{\tilde{t},[0.5,0.5]\} \\
& =\left\{\begin{array}{cll}
\tilde{t}, & \text { if } \tilde{t} \leq[0.5,0.5], \\
{[0.5,0.5]>[1,1]-\tilde{t},} & \text { if } \tilde{t}>[0.5,0.5],
\end{array}\right.
\end{aligned}
$$

so that $x, y \in \tilde{\mu}_{\tilde{t}} \cup Q(\tilde{\mu} ; \tilde{t})=[\tilde{\mu}]_{\tilde{t}}$. Next we suppose that $\tilde{\mu}(x y)+\tilde{t}>[1,1]$ and $\tilde{t}>[0.5,0.5]$, and from $\left(\mathrm{d}_{6}\right)$ we have $r \min \{\tilde{\mu}(x), \tilde{\mu}(y)\} \geq r \min \{\tilde{\mu}(x y),[0.5,0.5]\}$

$$
=\left\{\begin{array}{cll}
{[0.5,0.5]>[1,1]-\widetilde{t},} & \text { if } & \tilde{\mu}(x y) \geq[0.5,0 . \\
{[\tilde{\mu}(x y)>[1,1]-\widetilde{t},} & \text { if } & \tilde{\mu}(x y)<[0.5,0 .
\end{array}\right.
$$

and thus $x, y \in Q(\tilde{\mu} ; \tilde{t}) \subseteq[\tilde{\mu}]_{\tilde{t}}$. If $\tilde{t} \leq[0.5,0.5]$, then

$r \min \{\tilde{\mu}(x), \tilde{\mu}(y)\} \geq r \min \{\tilde{\mu}(x y),[0.5,0.5]\}$

$$
\begin{aligned}
& \geq r \min \{[1,1]-\tilde{t},[0.5,0.5]\} \\
& =[0.5,0.5] \\
& \geq \widetilde{t},
\end{aligned}
$$

Conversely, suppose that for all $\tilde{t} \in D(0,1]$, the set $[\tilde{\mu}]_{\tilde{t}}(\neq \phi)$ is a filter of $S$. If there exist $x_{0}, y_{0} \in S$ with $x_{0} \leq y_{0}$ such that $\tilde{\mu}\left(y_{0}\right)<r \min \left\{\widetilde{\mu}\left(x_{0}\right),[0.5,0.5]\right\}$,

then $\widetilde{\mu}\left(y_{0}\right)<\widetilde{t}_{0} \leq r \min \left\{\widetilde{\mu}\left(x_{0}\right),[0.5,0.5]\right\}$ for some $\tilde{t}_{0} \in D(0,0.5]$. It follows that $x_{0} \in \tilde{\mu}_{\tilde{t}_{0}} \subseteq[\tilde{\mu}]_{\widetilde{t}_{0}}$ and so $x_{0} \in[\widetilde{\mu}]_{\tilde{t}_{0}}$. Thus $\tilde{\mu}\left(x_{0}\right) \geq \widetilde{t}_{0}$ or $\tilde{\mu}\left(x_{0}\right)+\tilde{t}_{0}>[1,1]$. Since $y_{0} \geq x_{0} \in[\tilde{\mu}]_{\tilde{t}_{0}}$ and for $\tilde{t}_{0} \in D(0,0.5]$ , $[\tilde{\mu}]_{\tilde{t}}$ is a filter, we have $y_{0} \in[\tilde{\mu}]_{\tilde{t}_{0}}$. Hence $\tilde{\mu}\left(y_{0}\right) \geq \tilde{t}_{0}$ or $\tilde{\mu}\left(y_{0}\right)+\tilde{t}_{0}>[1,1], \quad$ a $\quad$ contradiction. Hence $\tilde{\mu}(y) \geq r \min \{\tilde{\mu}(x),[0.5,0.5]\}$ for all $x, y$ with $x \leq y$. If there exist $a, b \in S$ such that $\tilde{\mu}(a b)<r \min \{\tilde{\mu}(a), \tilde{\mu}(b),[0.5,0.5]\}$, then $\quad \tilde{\mu}(a b)<\widetilde{t_{1}} \leq r \min \{\tilde{\mu}(a), \tilde{\mu}(b),[0.5,0.5]\} \quad$ for $\quad$ some $\widetilde{t}_{1} \in D(0,0.5]$. Hence $a, b \in \widetilde{\mu}_{\widetilde{t}_{1}} \subseteq\left[\tilde{\mu}_{\tilde{t}_{1}}\right.$ and so $a, b \in\left[\tilde{\mu}_{\tilde{t}_{1}}\right.$ but $a b \bar{\in} \widetilde{\mu}_{\widetilde{t}_{1}}$ and $a b \bar{\in} Q\left(\tilde{\mu} ; \tilde{t}_{1}\right)$ i.e., $a b \bar{\epsilon}\left[\widetilde{\mu}_{\widetilde{t}_{1}}\right.$, a contradiction. Hence $\tilde{\mu}(x y) \geq r \min \{\tilde{\mu}(x), \tilde{\mu}(y),[0.5,0.5]\}$ for all $x, y \in S$. Finally, if there exist $\quad c, d \in S \quad$ such that $r \min \{\tilde{\mu}(c), \tilde{\mu}(d)\}<r \min \{\tilde{\mu}(c d),[0.5,0.5]\}, \quad$ then $r \min \{\tilde{\mu}(c), \tilde{\mu}(d)\}<\tilde{t}_{2} \leq r \min \{\tilde{\mu}(c d),[0.5,0.5]\} \quad$ for $\quad$ some $\tilde{t}_{2} \in D(0,0.5]$. Hence $c d \in \tilde{\mu}_{\tilde{t}_{2}} \subseteq\left[\tilde{\mu}_{\tilde{t}_{2}}\right.$. It follows that $c d \in\left[\tilde{\mu}_{\tilde{t}_{2}}\right.$ and hence $c, d \in[\tilde{\mu}]_{\widetilde{t}_{2}}$. Thus $\tilde{\mu}(c) \geq \widetilde{t}_{2}$ or $\tilde{\mu}(c)+\widetilde{t}_{2}>[1,1]$ and $\tilde{\mu}(d) \geq \tilde{t}_{2} \quad$ or $\quad \tilde{\mu}(d)+\tilde{t}_{2}>[1,1], \quad$ a contradiction. Thus $r \min \{\tilde{\mu}(x), \tilde{\mu}(y)\} \geq r \min \{\tilde{\mu}(x y),[0.5,0.5]\}$ for all $x, y \in S$. Thus $\tilde{\mu}$ is an interval valued $(\in, \in \vee \mathrm{q})$-fuzzy filter of $S$.

In (28], Yuan, Zhang and Ren gave the definition of a fuzzy subgroup with thresholds which is a generalization of Rosenfeld's fuzzy subgroup, and Bhakat and Das's fuzzy subgroup. Based on that definition (28], we extended the concept of a fuzzy subgroup with thresholds to the concept of an interval valued fuzzy filter with thresholds as follows.

Definition 4.12. Let $\tilde{r}, \tilde{s} \in D[0,1]$ and $\tilde{r}<\tilde{s}$. Let $\tilde{\mu}$ be an interval valued fuzzy subset of an ordered semigroup $S$. Then $\tilde{\mu}$ is called an interval valued fuzzy filter with thresholds $(\widetilde{r}, \widetilde{S}]$ of $S$ if it satisfies the following assertions:

$\left(\mathrm{e}_{1}\right)(\forall x, y \in S) r \max \{\widetilde{\mu}(y), \widetilde{r}\} \geq r \min \{\widetilde{\mu}(x), \widetilde{s}\}$ with $x \leq y$

$\left(\mathrm{e}_{2}\right)(\forall x, y \in S) \quad r \max \{\tilde{\mu}(x y), \tilde{r}\} \geq r \min \{\tilde{\mu}(x), \tilde{\mu}(y), \widetilde{s}\}$,

$\left(\mathrm{e}_{3}\right)(\forall x, y \in S) r \max \{\tilde{\mu}(x), \tilde{\mu}(y), \widetilde{r}\} \geq r \min \{\tilde{\mu}(x y), \widetilde{s}\}$.

If $\tilde{\mu}$ is an interval valued fuzzy filter of $S$ with thresholds of $S$, then we concluded that $\tilde{\mu}$ is an ordinary interval valued fuzzy filter when $\tilde{r}=[0,0], \widetilde{s}=[1,1]$ and $\tilde{\mu}$ is an interval valued $(\in, \in \vee \mathrm{q})$ fuzzy filter when $\widetilde{r}=[0,0], \widetilde{s}=[0.5,0.5]$. Now, we characterize interval valued fuzzy filters with thresholds $(\widetilde{r}, \widetilde{S}]$ of $S$, by their level filters.

Theorem 4.13. An interval valued fuzzy subset $\tilde{\mu}$ of an ordered semigroup $S$ is an interval valued fuzzy filter with thresholds $(\widetilde{r}, \widetilde{S}]$ of $S$ if and only if $\tilde{\mu}_{\tilde{t}}(\neq \phi)$ is a filter of $S$ for all $\tilde{t} \in D(\widetilde{r}, \widetilde{s}]$.

Proof. Let $\tilde{\mu}$ be an interval valued fuzzy filter with thresholds $(\widetilde{r}, \widetilde{s}]$ of $S$ and $\tilde{t} \in D(\widetilde{r}, \widetilde{s}]$. Let $x, y \in S$ with $x \leq y$. Let $x \in \tilde{\mu}_{\tilde{t}}$ , then $\tilde{\mu}(x) \geq \tilde{t}$ and from $\left(\mathrm{e}_{1}\right)$ of Definition 4.12, it follows that

and so $x, y \in \tilde{\mu}_{\tilde{t}} \subseteq[\tilde{\mu}]_{\tilde{t}}$. Consequently, $[\tilde{\mu}]_{\tilde{t}}$ is a filter of $S$.

Copyright $(C 2018$ Authors. This is an open access article distributed under the Creative Commons Attribution License, which permits unrestricted use, distribution, and reproduction in any medium, provided the original work is properly cited. 
$r \max \{\widetilde{\mu}(y), \widetilde{r}\} \geq r \min \{\widetilde{\mu}(x), \widetilde{s}\}$

$$
\begin{aligned}
& \geq r \min \{\tilde{t}, \widetilde{s}\} \\
& \geq \tilde{t}>\widetilde{r},
\end{aligned}
$$

So $\widetilde{\mu}(y) \geq \tilde{t}$ and hence $y \in \tilde{\mu}_{\tilde{t}}$. Let $x, y \in \tilde{\mu}_{\tilde{t}}$, then $\widetilde{\mu}(x) \geq \tilde{t}$ , and by $\left(\mathrm{e}_{2}\right)$, we have

$$
\begin{aligned}
r \max \{\widetilde{\mu}(x y), \widetilde{r}\} & \geq r \min \{\widetilde{\mu}(x), \widetilde{\mu}(y), \widetilde{s}\} \\
& \geq r \min \{\tilde{t}, \widetilde{s}\} \\
& \geq \widetilde{t}>\widetilde{r},
\end{aligned}
$$

and hence $\tilde{\mu}(x y) \geq \tilde{t}$, implies that $x y \in \tilde{\mu}_{\tilde{t}}$. For $x y \in \tilde{\mu}_{\tilde{t}}$, we have $\tilde{\mu}(x y) \geq \tilde{t}$ and by $\left(\mathrm{e}_{3}\right)$, it follows that

$$
\begin{aligned}
r \max \{\widetilde{\mu}(x), \widetilde{\mu}(y), \widetilde{r}\} & \geq r \min \{\widetilde{\mu}(x y), \widetilde{s}\} \\
& \geq r \min \{\widetilde{t}, \widetilde{s}\} \\
& \geq \widetilde{t}>\widetilde{r}
\end{aligned}
$$

and so $\tilde{\mu}(x) \geq \tilde{t}, \tilde{\mu}(y) \geq \tilde{t}$ that is, $x, y \in \tilde{\mu}_{\tilde{t}}$.

Conversely, let $\tilde{\mu}$ be an interval valued fuzzy subset of $S$ such that $\tilde{\mu}_{\tilde{t}}(\neq \phi)$ is a filter of $S$ for all $\tilde{t} \in D(\widetilde{r}, \widetilde{s}]$. If there exist $x, y \in S \quad$ with $\quad x \leq y \quad$ such that $r \max \{\tilde{\mu}(y), \widetilde{r}\}<r \min \{\tilde{\mu}(x), \widetilde{s}\}=\widetilde{t}$, then $\quad \tilde{t} \in D(\widetilde{r}, \widetilde{s}]$, $\tilde{\mu}(y)<\tilde{t}$ and $x \in \tilde{\mu}_{\tilde{t}}$. Since $y \geq x \in \widetilde{\mu}_{\tilde{t}}$ and $\tilde{\mu}_{\tilde{t}}$ is a filter of $S$, we have $y \in \tilde{\mu}_{\tilde{t}}$. Then $\tilde{\mu}(y) \geq \tilde{t}$, a contradiction. Hence $r \max \{\tilde{\mu}(x), \tilde{\mu}(y), \tilde{r}\} \geq r \min \{\tilde{\mu}(x y), \tilde{s}\}$ for all $x, y \in S$ with $x \leq y$. If there exist $x, y \in S$ such that

$$
r \max \{\tilde{\mu}(x y), \widetilde{r}\}<r \min \{\widetilde{\mu}(x), \widetilde{\mu}(y), \widetilde{s}\}=\widetilde{t},
$$

then $\tilde{t} \in D(\tilde{r}, \tilde{s}], \tilde{\mu}(x y)<\tilde{t}$ and $x_{\tilde{t}} \in \tilde{\mu}, y_{\tilde{t}} \in \tilde{\mu}$. Since $\tilde{\mu}_{\tilde{t}}$ is a filter of $S$ and $x_{\tilde{t}} \in \tilde{\mu}, y_{\tilde{t}} \in \tilde{\mu}$, we have $(x y)_{\min \{\tilde{t}, \tilde{t}\}}=(x y)_{\tilde{t}} \in \tilde{\mu}$, then $\tilde{\mu}(x y) \geq \tilde{t}$, a contradiction. Hence $r \max \{\widetilde{\mu}(x y), \widetilde{r}\} \geq r \min \{\widetilde{\mu}(x), \widetilde{\mu}(y), \widetilde{s}\}$ for all $x, y \in S$. In a similar way we can prove that $r \max \{\tilde{\mu}(x), \tilde{\mu}(y), \widetilde{r}\} \geq r \min \{\widetilde{\mu}(x y), \widetilde{s}\} \quad$ for $\quad$ all $\quad x, y \in S$. Therefore $\widetilde{\mu}$ is a fuzzy filter of $S$.

\section{Remark 4.14.}

(1). By Definition 4.12, we have the following conclusion: if $\tilde{\mu}$ is an interval valued fuzzy filter with thresholds $(\widetilde{r}, \widetilde{s}]$ of $S$, then we have:

(i) $\widetilde{\mu}$ is an ordinary interval valued fuzzy filter when $\widetilde{r}=[0,0], \widetilde{s}=[1,1]$,

(ii) $\tilde{\mu}$ is an interval valued $(\in, \in \vee \mathrm{q})$-fuzzy filter when $\widetilde{r}=[0,0], \widetilde{s}=[0.5,0.5]$.

(2). By Definition 4.12, we can define other kinds of interval valued fuzzy filters of $S$, such as interval valued fuzzy filter with thresholds $([0.5,0.5],[0.6,0.6]]$ and $([0.4,0.4],[0.8,0.8]]$ of $S$, etc.

(3). However, the interval valued fuzzy filters with thresholds of $S$ may not be an ordinary interval valued fuzzy filter and may not be an interval valued $(\in, \in \vee q)$-fuzzy filter as shown in the following example:

Example 4.15. Consider the ordered semigroup as given in Example 4.2 and define an interval valued fuzzy subset $\tilde{\mu}$ as follows:

$$
\tilde{\mu}(x)=\left(\begin{array}{ccccc}
a & b & c & d & e \\
{[0.6,0.7]} & {[0.4,0.5]} & {[0.3,0.4]} & {[0.7,0.8]} & {[0.8,0.9]}
\end{array}\right.
$$

Then

$$
\widetilde{\mu}_{\tilde{t}}=\left\{\begin{array}{ccc}
S, & \text { if } & {[0,0]<\tilde{t} \leq[0.3,0.4],} \\
\{a, d, e\}, & \text { if } & {[0.5,0.6]<\tilde{t} \leq[0.6,0.7],} \\
\{a, d, e, f\}, & \text { if } & {[0.4,0.5]<\tilde{t} \leq[0.5,0.6],} \\
\{a, b, d, e, f\}, & \text { if } & {[0.3,0.4]<\tilde{t} \leq[0.4,0.5],} \\
\phi, & \text { if } & {[0.8,0.9]<\tilde{t} \leq[1,1] .}
\end{array}\right.
$$

Thus, $\tilde{\mu}$ is an interval valeud fuzzy filter with thresholds $([0.5,0.6],[0.6,0.7]]$ of $S$ but not an interval valued fuzzy filter and an interval valued $(\in, \in \vee \mathrm{q})$-fuzzy filter of $S$.

\section{References}

1 S. K. Bhakat, P. Das, $(\in, \in \vee q)_{\text {-fuzzy subgroups, Fuzzy Sets and }}$ Systems, 80 (1996) 359-368.

2 S. K. Bhakat, P. Das, Fuzzy subrings and ideals redefined, Fuzzy Sets and Systems, 81 (1996) 383-393.

3 B. Davvaz, O. Kazanc and S. Yamak, Generalized fuzzy n-ary subpolygroups endowed with interval valued membership functions, Journal Intelligent and Fuzzy Systems, 20 (4-5) (2009) 159-168.

4 B. Davvaz and $Z$. Mozafar, $(\in, \in \vee q)$-fuzzy Lie subalgebra and ideals, International Journal of Fuzzy Systems, 11(2) (2009) 123-129.

5 B. Davvaz and P. Corsini, On $(\alpha, \beta)$-fuzzy $H_{v \text {-ideals of }} H_{v \text {-rings, }}$ Iran. J. Fuzzy Syst., 5(2) (2008) 35-47.

6 B. Davvaz, J. Zhan, K.P. Shum, Generalized fuzzy polygroups endowed with interval valued membership functions, Journal of Intelligent and Fuzzy Systems, 19(3) (2008) 181-188.

7 B. Davvaz, Fuzzy R-subgroups with thresholds of near-rings and implication operators, Soft Computing, 12 (2008) 875-879.

8 B. Davvaz, $(\in, \in \vee q)_{\text {-fuzzy subnear-rings and ideals, Soft Computing, }}$ 10 (2006) 206-211.

9 Y. B. Jun, A. Khan and M. Shabir, Ordered semigroups characterized by

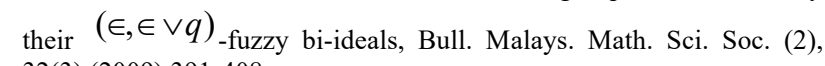
32(3) (2009) 391-408.

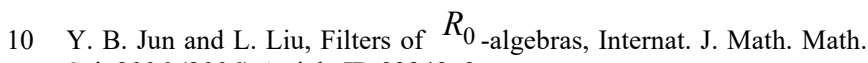
Sci. 2006 (2006) Article ID 93249, 9 pages.

11 Y. B. Jun and S. Z. Song, Generalized fuzzy interior ideals in semigroups, Information Sciences, 176 (2006) 3079-3093.

12 O. Kazanci and S. Yamak, Generalized fuzzy bi-ideals of semigroup, Soft Comput, 12 (2008) 1119-1124.

13 O. Kazanci and B. Davvaz, Fuzzy n-ary polygroups related to fuzzy points, Computers \& Mathematics with Applications, 58(7) (2009) 14661474.

14 N. Kehayopulu and M. Tsingelis, Fuzzy sets in ordered groupoids, Semigroup Forum, 65 (2002) 128-132.

15 A. Khan and M. Shabir, $(\alpha, \beta)$-fuzzy interior ideals in ordered semigroups, Lobachevskii Journal of Mathematics, 30(1) (2009) 3039.

16 B. Davvaz and A. Khan, Characterizations of regular ordered semigroups in terms of $(\alpha, \beta)$-fuzzy generalized bi-ideals, Inform. Sci. 181 (2011) $1759-1770$.

17 A. Khan, Y. B. Jun, N. H. Sarmin and F. M. Khan, Ordered semigroups characterized by $\left(\in, \in \vee q_{k}\right)_{\text {-fuzzy }}$ generalized bi-ideals, Neural Computing and Applications (2011) DOI: 10.1007/s00521-011-0731-2.

18 A. Khan, Y. B. Jun and M. Shabir, Ordered semigroups characterized by interval valued $(\in, \in \vee q)_{\text {-fuzzy bi-ideals, to appear in Journal of }}$ Intelligent and Fuzzy Systems.

19 Y. B. Jun, A. Khan and M. Shabir, Ordered semigroups characterized by their $(\in, \in \vee q)_{\text {-fuzzy bi-ideals, Bull. Malays. Math. Sci. Soc. (2) } 32}$ (3) (2009) 391-408.

20 N. Kuroki, On fuzzy ideals and fuzzy bi-ideals in semigroups, Fuzzy Sets and Systems, 5 (1981) 203-215.

21 S. K. Lee and K. Y. Park, On right (left) duo po-semigroups, KangweonKyungki Math. Jour., 11(2) (2003) 147-153.

22 L. Liu and K. Li, Fuzzy implicative and Boolean filters of $R_{0}$-algebras, Information Sciences, 171 (2005) 61-71. 
23 X. Ma, J. Zhan and Y. B. Jun, On $(\in, \in \vee q)_{\text {fuzzy filters of }} R_{0}$ algebras, Math. Log. Quart., 55 (2009) 493-508.

24 J. N. Mordeson, D. S. Malik and N. Kuroki, Fuzzy Semigroups, Studies in Fuzziness and Soft Computing Vol. 131, Springer-Verlag Berlin (2003).

25 P. M. Pu and Y. M. Liu, Fuzzy topology I, neighborhood structure of a fuzzy point and Moore-Smith convergence, J. Math. Anal. Appl., 76 (1980) 571-599.

26 A. Rosenfeld, Fuzzy groups, J. Math. Anal. Appl., 35 (1971) 512-517.

27 M. Shabir and A. Khan, Characterizations of ordered semigroups by the properties of their fuzzy generalized bi-ideals, New Mathematics and Natural Computation, 4(2) (2008) 237-250.

28 X. Yuan, C. Zhang and Y. Ren, Generalized fuzzy groups and manyvalued implications, Fuzzy Sets and Systems, 138 (2003) 205-211.

29 L.A. Zadeh, The concept of a linguistic variable and its application to approximate reason, Inform. Control 18 (1975) 199-249.

30 W. Zeng, H. Li, Relationship between similarity measure and entropy of interval valued fuzzy set, Fuzzy Sets Syst. 157 (2006) 1477-1484. 